\title{
NOUVELLE
}

\section{Cellules souches et réparation tubulaire après ischémie rénale}

Jean-Philippe Haymann, Laurent Baud
Inserm U.702 et Service d'Explorations fonctionnelles multidisciplinaires, Hôpital Tenon, 4, rue de la Chine, 75020 Paris, France.

laurent.baud@

tnn.ap-hop-paris.fr
> L'ischémie rénale est une cause majeure d'insuffisance rénale aiguë et de retard dans la reprise de fonction du rein transplanté [1]. Elle entraîne initialement des lésions de l'épithélium tubulaire, qui prédominent dans la médullaire externe, au niveau de la branche large ascendante de l'anse de Henle et surtout du segment S3 du tubule proximal. Surviennent d'abord des altérations du cytosquelette et une perte de la polarité des cellules épithéliales qui conduisent à une redistribution de protéines membranaires comme les molécules d'adhérence et la $\mathrm{Na}^{+}, \mathrm{K}^{+}$-ATPase. Les conséquences sont une disparition de la bordure en brosse, une diminution de la réabsorption de $\mathrm{Na}^{+}$et un détachement des cellules de la membrane basale. Si la période d'ischémie se prolonge, les cellules épithéliales meurent par apoptose ou nécrose. La réparation de ces lésions épithéliales implique la mobilisation et la prolifération de cellules «souches» ou progénitrices résidentes, et éventuellement de cellules souches circulantes, originaires principalement de la moelle osseuse (Figure 1).

\section{Présence et localisation de cellules souches dans le rein}

Des cellules «souches» résidentes ont été identifiées chez l'adulte non seulement dans des organes dont le renouvellement cellulaire est constant, comme la peau, la moelle osseuse ou l'intestin, mais aussi dans d'autres organes dont le renouvellement cellulaire est lent, comme le système nerveux central ou le rein [2]. Ces cellules doivent être capables de se renouveler et de se différencier dans tous les types cellulaires nécessaires. C'est principalement sur la base du critère de renouvellement lent que des cellules «souches » ou progénitrices ont été mises en évidence dans le rein. Dans ce but, des rats ont reçu pendant une brève période de temps de la bromodésoxyuridine (BrdU), un nucléotide qui s'incorpore dans I'ADN lors de sa synthèse. Après un délai de plusieurs jours ou semaines, les reins de ces rats ont été prélevés et les cellules rénales, marquées par le BrdU, identifiées par une technique d'immunofluorescence: ce sont des cellules capables de proliférer (le BrdU a été incorporé dans I'ADN lors de sa synthèse), mais dont le renouvellement est lent (le BrdU n'a pas été dilué par de multiples divisions cellulaires). Une première étude [3] a montré que ces cellules sont localisées principalement dans les tubules proximaux, à un moindre degré dans les canaux collecteurs, et à un très faible degré dans la branche large ascendante des anses de Henle et dans les tubules distaux. Elles sont absentes dans les glomérules et les vaisseaux. Une étude plus récente [4] a montré, et c'est inattendu, que les cellules marquées par le BrdU sont particulièrement nombreuses dans les canaux collecteurs et l'interstitium de la papille, à proximité des vaisseaux. Cependant, l'implication réelle de ces cellules de la papille dans la réparation des lésions ischémiques tubulaires n'a pas encore été démontrée.

Les gènes exprimés spécifiquement par les cellules «souches » ou progénitrices rénales sont encore mal définis. On sait que dans la moelle osseuse, les cellules souches hématopoïétiques expriment le gène qui code pour la protéine de transport ABCG2. Cette expression, qui définit le caractère marginal des cellules souches (side population), leur permet d'exclure des marqueurs colorés comme le Hoechst 33342 et des agents cytotoxiques, augmentant ainsi leur chance de survie. Dans le rein adulte, 0,03\% à $0,1 \%$ des cellules excluent le Hœchst 33342 [5]. Alors que ces cellules ont la capacité de se différencier en cellules des lignées hématopoïétiques et non hématopoïétiques, elles ne semblent pas servir de précurseurs aux cellules

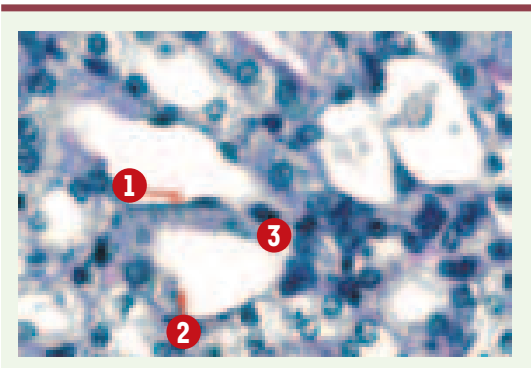

Figure 1. Participation de cellules résidentes et circulantes à la réparation de l'épithélium dans le tubule proximal après ischémie rénale. Étalement, migration et prolifération des cellules épithéliales survivantes (1). Contribution éventuelle des cellules progénitrices circulantes et/ou interstitielles grâce à leur capacité de différenciation épithéliale (2). Contribution éventuelle des cellules mésenchymateuses circulantes et/ou interstitielles grâce à leur capacité de sécrétion paracrine de facteurs de croissance ou de survie (3). 
tubulaires. Dans le rein adulte, 0,8\% à $1,2 \%$ des cellules expriment CD 133, un autre marqueur des cellules souches hématopoïétiques [6]. Ces cellules, qui sont dispersées dans l'interstitium, ont la capacité de proliférer et de se différencier en cellules épithéliales formant in vivo des structures tubulaires.

\section{Repopulation du rein transplanté par} des cellules souches hématopoiiétiques L'hypothèse selon laquelle des cellules souches circulantes participent aussi à la réparation tubulaire a été testée initialement en recherchant la présence de cellules porteuses d'un chromosome y dans le rein transplanté, le donneur du transplant étant féminin et le receveur masculin [7]. Les résultats ont montré que, dans ces conditions, $1 \%$ à $10 \%$ des cellules de localisation et de phénotype tubulaire sont positives pour le chromosome $y$, et proviennent donc de la circulation du sujet receveur. Cette repopulation du rein transplanté ne survient qu'en cas de nécrose tubulaire aiguë liée à la phase d'ischémie. Confirmation et précisions ont été obtenues ensuite grâce à des modèles expérimentaux d'ischémie rénale. Dans un premier modèle, des souris irradiées ont été greffées avec des cellules médullaires provenant de souris transgéniques $l a c Z$, c'est-à-dire de cellules détectables grâce à leur forte activité $\beta$-galactosidase [8]. Après plusieurs semaines nécessaires à la reconstitution de la moelle, l'un des deux reins de ces souris a été soumis à 25 minutes d'ischémie et 7 jours de reperfusion. Dans le rein ischémique uniquement, environ $20 \%$ des cellules de la médullaire externe apparaissent avec une forte activité $\beta$-galactosidase et expriment la mégaline, un marqueur de différenciation des cellules épithéliales du tubule proximal, suggérant une participation significative des cellules de la moelle osseuse à la régénération tubulaire. Ces cellules de la moelle osseuse ne sont pas des cellules déjà engagées dans la différenciation hématopoïétique, mais des cellules souches hématopoïétiques (elles expriment le stem-cell antigen-1 et $c$-kit). Les mêmes résultats ont été obtenus en injectant les cellules de la moelle osseuse au moment même où les souris sont soumises à une ischémie rénale [8].

Dans leur ensemble, les résultats obtenus sont donc en faveur d'une participation des cellules souches hématopoïétiques à la régénération de l'épithélium tubulaire après ischémie et conduisent à proposer la mobilisation de ces cellules comme nouvelle stratégie thérapeutique dans l'insuffisance rénale aiguë. Malheureusement, plusieurs études très récentes limitent ces perspectives. D'abord, les résultats obtenus avec les souris transgéniques lacZ pourraient avoir été largement surestimés, les cellules tubulaires des souris sauvages montrant elles aussi une forte activité $\beta$-galactosidase. Ensuite, la mobilisation des cellules souches hématopoïétiques à partir de la moelle risque de s'accompagner de la mobilisation de cellules inflammatoires, entraînant l'aggravation plutôt que l'amélioration attendue de l'insuffisance rénale aiguë [9].

\section{Effet protecteur paracrine des cellules} souches mésenchymateuses

Les cellules souches hébergées dans la moelle osseuse incluent non seulement des cellules souches hématopoïétiques, mais aussi des cellules souches mésenchymateuses, qui ont un potentiel de différenciation en cellules nerveuses, musculaires et hépatiques. Une étude très récente du groupe de $C$. Westenfelder (Salt Lake City, États-Unis) démontre que l'injection intra-artérielle de ces cellules immédiatement ou 24 heures après un épisode d'ischémie rénale chez le rat entraîne une protection [10]. Celle-ci se traduit par une amélioration de la fonction rénale et une diminution des lésions histologiques tubulaires. Pourtant, les cellules souches mésenchymateuses injectées ne se différencient pas en cellules épithéliales. Grâce à une technique très intéressante de microscopie confocale biphotonique in vivo, les auteurs montrent qu'elles n'intègrent pas la structure tubulaire, mais restent attachées à l'endothélium des capillaires péritubulaires. C'est là qu'elles exercent un effet protecteur paracrine, en libérant des facteurs anti-apoptotiques ou mitogènes et des cytokines anti-inflammatoires.

Cette «hypothèse paracrine » explique aussi la protection apportée par les cellules souches mésenchymateuses dans les lésions ischémiques myocardiques [11]. Elle pourrait en fait expliquer le rôle attribué aux cellules souches médullaires dans bien des modèles de régénération/réparation. $\diamond$

What is the contribution of stem cells to tubular repair after renal ischemia?

\section{RÉFÉRENCES}

1. Schrier RW, Wang W, Poole B, Mitra A. Acute renal failure: definitions, diagnosis, pathogenesis, and therapy. J Clin Invest 2004 ; $114: 5$-14.

2. Oliver JA. Adult renal stem cells and renal repair. Curr Opin Nephrol Hypertens 2004 ; 13 : 17-22.

3. Maeshima A, Yamashita S, Nojima Y. Identification of renal progenitor-like tubular cells that participate in the regeneration process of the kidney. J Am Soc Nephrol 2003 ; 14 : 3138-46.

4. Oliver JA, Maarouf 0 , Cheema FH, et al. The renal papilla is a niche for adult kidney stem cells. J Clin Invest $2004 ; 114: 795-804$.

5. Iwatani H, Ito T, Imai $\varepsilon$, et al. Hematopoietic and nonhematopoietic potentials of Hoechst ${ }^{\text {low }} /$ side population cells isolated from adult rat kidney. Kidney Int 2004 ; 65 : 1604-14.

6. Bussolati B, Bruno S, Grange $C$, et al. Isolation of progenitor cells from adult human kidney. Am J Pathol $2005 ; 166: 545-55$.

7. Gupta S, Verfaillie C, Chmielewski D, et al. A role for extrarenal cells in the regeneration following acute renal failure. Kidney Int $2002 ; 62$ : 1285-90.

8. Kale S, Karihaloo A, Clark PR, et al. Bone marrow stem cells contribute to repair of the ischemically injured renal tubule.J Clin Invest $2003 ; 112: 42-9$.

9. Tögel F, Isaac J, Westenfelder C. Hematopoietic stem cell mobilization-associated granulocytosis severely worsens acute renal failure. J Am Soc Nephrol 2004 ; $15: 1261-7$.

10. Tögel F, Hu Z, Weiss K, et al. Administered mesenchymal stem cells protect against ischemic acute renal failure through differentiation-independent mechanisms. Am J Physiol Renal Physiol 2005 (sous presse).

11. Gnecchi M, He H, Liang OD, et al. Paracrine action accounts for marked protection of ischemic heart by Akt-modified mesenchymal stem cells. Nat Med 2005; $11: 367-8$. 